\title{
A Systematic Approach to Modeling Organic Rankine Cycle Systems for Global Optimization
}

\author{
Vathna $\mathrm{Am}^{1}$, Jonathan Currie ${ }^{2}$ and David I. Wilson ${ }^{3}$
}

\begin{abstract}
The demand for organic Rankine cycle (ORC) systems to be efficient and economically competitive drives the need for a reliable and robust modeling approach that is suitable for optimization. However existing commercial simulation software is not typically tailored for optimization and they generally cannot guarantee global optimum. This paper proposes a modeling approach to approximate a rigorous simulation model that is suitable for global optimization. This involves a combination of regression and thermodynamic analysis, in addition to integer programming techniques. Three different solvers, COBYLA, SCIP, and BARON are used to optimize the ORC model and are compared against each other to demonstrate the prospect of achieving the global optimum using this approach. In addition, this paper also presents a technique to improve the model accuracy by using a piecewise fit to approximate the output characteristic of the ORC unit operations.
\end{abstract}

\section{INTRODUCTION}

With the inevitability of the world's non-renewable resources becoming scarcer and the progressive pressure for the power generation sector to reduce greenhouse gases emission and pollutants, there has been a massive growth in renewable power generation technologies. Geothermal energy is one example of a reliable source of sustainable energy. As of 2010, there are 24 countries that have used geothermal energy for electricity generation [1]. Of all the geothermal power plants (GPP), organic Rankine cycle (ORC) power plants are the most common type of geothermal power plants in the world with 203 units in operation as of December 2014 , constituting to over $35 \%$ of all geothermal units [2].

Given the large number of ORC systems around the world and the potential for more to be installed due to the relatively abundant low-temperature geothermal resources, there is a considerable amount of literature on ORC modeling and optimization. Often these ORC plants are simulated using the sequential modular approach, via a standard modeling software such as Aspen Plus [3] or GateCycle [4], and then are subsequently optimized using heuristic methods or sophisticated algorithms [5], [6].

However, one major concern with using this approach lies in the robustness and efficiency of the model when it is used in optimization problems. Therefore, this paper will discuss the limitations of sequential modular models and propose

\footnotetext{
${ }^{1}$ School of Engineering, Computer \& Mathematical Sciences, Auckland University of Technology, Auckland, New Zealand; e-mail: vameaut.ac.nz

${ }^{2}$ School of Engineering, Computer \& Mathematical Sciences, Auckland University of Technology, Auckland, New Zealand

${ }^{3}$ Industrial Information \& Control Centre, Auckland University of Technology, Auckland, New Zealand
}

a modeling approach for ORC systems that is intended for efficient global optimization.

\section{Sequential Modular Model and EQUATION-ORIENTED MODEL}

Traditionally, GPPs are often modeled using the sequential modular (SM) approach where unit operation modules (or functions) are linked together in the flow order through the plant. The output of each module is calculated from the output of the previous module in the flowsheet; therefore, the stream information, i.e. mass flows, temperatures, enthalpies, etc., will propagate from the beginning of the plant's process through to the end of the flowsheet.

However, there are some key limitations to using the SM approach, especially when dealing with optimization problems. Many of the derivative optimization solvers, like fmincon (MATLAB's solver) [7] or IPOPT [8], that require the first and/or second derivatives of the objective function and constraints, will struggle to find accurate derivatives from SM models due to complex thermodynamics and/or internal model iterations. Consequently, the derivatives are approximated using finite differences leading to various optimization problems such as convergence issues and long computation times [9]. Furthermore, it is recognized that optimization problems are solved more accurately and efficiently if the analytical derivative functions are provided, [10], [11]. In addition, solvers that are compatible with the SM model structure cannot guarantee that the optimum found is the global optimum, which might be a disadvantage in today's competitive market.

An alternative method is to use the equation-oriented (EO) approach, where systems are represented as a set of equations that are solved simultaneously. In contrast, to the SM approach, EO approach allows standard optimization problems to be formulated relatively efficiently from the model structure, and the mass and energy balance equations are solved simultaneously with the optimization problem [12]. In addition, provided that if all the equations are algebraic and analytically differentiable, some global optimizers (white-box optimizers) can exploit this algebraic structure and analytically calculate the required derivatives for optimization. This means that the numerical issues discussed above can be bypassed or reduced. Thus, this paper aims to demonstrate how an ORC system can be modeled and optimized using an algebraic EO approach and using whitebox global solvers, namely SCIP [13] and BARON [14], to deterministically find the global optimum of the plant. 
Furthermore, the results will be compared with a blackbox optimizer, namely COBYLA [15] (supplied by NLopt [16]), that cannot guarantee global optimality to highlight the difference between the two optimization techniques. The novelty of this research is the application of the proposed modeling framework to an ORC system to address the issues associated with the conventional SM optimization approach and to provide an efficient model that is tailored for global optimization.

\section{Modeling An ORC System}

This paper illustrates the proposed modeling approach using a simple four-unit-operation ORC system. Fig. 1 shows the process flow diagram (PFD) of the ORC system, which consists of a cycle including a turbine, condenser, feed pump, and an evaporator. The topology of this ORC system has been analyzed extensively in many academic papers [17]-[19] and in many standard thermodynamic textbooks such as [2], [20].

\section{A. Model Description}

The specific ORC system described in [19] was used to demonstrate the proposed modeling approach with plant parameters labeled in Fig. 1. Since this research focuses mainly on the modeling and optimization aspect of an ORC system, and not the effect of different working fluids, only R227ea (1, 1, 1, 2, 3, 3, 3-Heptafluoropropane) was used due to its favorable thermodynamic properties.

In addition, the ORC system was assumed as a steadystate and steady-flow process; whereby changes in kinetic and potential energy were neglected, and losses induced by friction were neglected. The thermodynamic and transport properties of the brine were considered to be the same as water; chemical substances and non-condensible gases were neglected. Furthermore, the pressure drops across heat exchangers and pipelines were neglected.

\section{B. High Fidelity SM Model}

The high fidelity SM model in this research was constructed using JSteam MATLAB Toolbox v1.70 [21]. The construction of the model used unit operation function calls

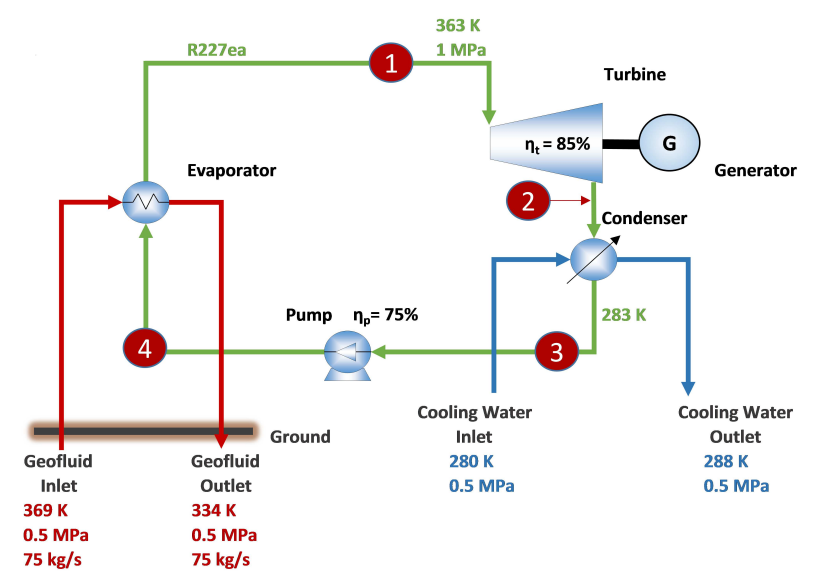

Fig. 1. The process flow diagram of a basic organic Rankine cycle system. and the current REFPROP (version 9.1) thermodynamic package [22], which were considered as the "gold standard" for the purposes of this study.

As previously mentioned, these high fidelity SM models are not tailored for optimization. Therefore, this model was not built to be optimized but instead was used to validate the approximate EO model since it provides a very accurate representation of the ORC system. This is an important part of this proposed modeling framework, as it will show the reliability and accuracy of the approximate EO model to the original system. Once the model is constructed and solved using a nonlinear system solver (such as fsolve in MATLAB), it can be used as initial guess for the optimization problem discussed in Section III-C. Since different initialization values can result in different optimization times and solutions, it is important that a feasible initial guess is used for the optimization problem. Therefore, for this research, the initialization values were taken from the base case scenario [19] that was solved in the SM model to minimize the issues associated with a poor initial guess.

In order to keep this paper concise, the construction of this model will not be discussed but a similar system is detailed in the tutorial section of the JSteam software, [21].

\section{Approximate EO Model}

As mentioned in Section II, in order to utilize SCIP and BARON solvers, an algebraic description of the ORC model is required. The task of constructing the $\mathrm{EO}$ model of an ORC systems amounts to deriving a set of deterministic algebraic equations describing the process of the system and approximating the output characteristic of the unit operations using regression and thermodynamic analysis. The following subsections will discuss how this model was constructed and the compromise between the accuracy of the model and the computational complexity of the optimization problem.

1) Objective Function: For this optimization problem, the objective function was to maximize the gross power output of the plant, which is defined as

$$
j=\left(h_{1}-h_{2}\right) \dot{m}_{1}
$$

where $h$ is the enthalpy value and $\dot{m}$ is the mass flow rate of the working fluid associated with the turbine in Fig. 1.

Note that this model is not limited to the gross power output; the objective function can be the net power output, the mass flow of the working fluid, or even a weighted economic analysis of the plant.

2) Mass and Energy Balance: The first set of equations is the mass and energy balance equations, which can be derived based on the first law of thermodynamics. Referring to Fig. 1, the following mass and energy balance equations are associated with the numbers labeled on the diagram, where $\dot{m}$ is the mass flow rate, $h$ is the enthalpy value, $\dot{W}_{\text {pump }}$ is the pump input power, $\dot{W}_{\text {turb }}$ is the turbine output power, 
$\mathrm{B}$ is the brine and $\mathrm{CW}$ is the cooling water.

$$
\begin{aligned}
& \dot{m}_{1}-\dot{m}_{2}=0 \\
& \dot{m}_{2}-\dot{m}_{3}=0 \\
& \dot{m}_{3}-\dot{m}_{4}=0 \\
& \dot{m}_{4}-\dot{m}_{1}=0 \\
& \dot{m}_{1} h_{1}-\dot{W}_{\text {turb }}-\dot{m}_{2} h_{2}=0 \\
& \dot{m}_{\mathrm{CW}_{\text {in }}} h_{\mathrm{CW}_{\text {in }}}+\dot{m}_{2} h_{2}-\dot{m}_{\mathrm{CW}_{\text {out }}} h_{\mathrm{CW}_{\text {out }}}-\dot{m}_{3} h_{3}=0 \\
& \dot{m}_{3} h_{3}+\dot{W}_{\text {pump }}-\dot{m}_{4} h_{4}=0 \\
& \dot{m}_{\mathrm{B}_{\text {in }}} h_{\mathrm{B}_{\text {in }}}+\dot{m}_{4} h_{4}-\dot{m}_{\mathrm{B}_{\text {out }}} h_{\mathrm{B}_{\text {out }}}-\dot{m}_{1} h_{1}=0
\end{aligned}
$$

From the fixed parameters mentioned in Section III-A, the following constants were calculated using REFPROP: $\dot{m}_{\mathrm{B}_{\text {in }}} h_{\mathrm{B}_{\text {in }}}$ is $30144 \mathrm{~kJ} / \mathrm{kg}, \dot{m}_{\mathrm{B}_{\text {ou }}} h_{\mathrm{B}_{\text {out }}}$ is $19133 \mathrm{~kJ} / \mathrm{kg}, h_{\mathrm{CW}_{\text {in }}}$ is $29.288 \mathrm{~kJ} / \mathrm{kg}, h_{\mathrm{CW}_{\text {out }}}$ is $62.832 \mathrm{~kJ} / \mathrm{kg}$. Note that while there is enough information to calculate $h_{1}$, it was intentionally kept as a decision variable in order to show how the turbine unit operation can be approximated using this modeling approach (see Section III-C.4). All the other variables in (2) and (3) are decision variables and will be calculated by the optimizer.

3) Operational Constraints: In addition to the mass and energy balance equations, there are operational constraints to consider. These are subcooling requirements, the pressure drop across heat exchangers, heat loss, etc. However, in compliance with [19] specifications and assuming that state 3 can operate between $283 \mathrm{~K}$ and the saturated temperature at $0.6 \mathrm{MPa}$, only the state of the working fluid entering the pump was of concern for this example, which needs to be greater or equal to $283 \mathrm{~K}$.

Equation (4)

$$
h_{3} \geq h_{f @ 283 \mathrm{~K}}
$$

ensures that state 3 will always be greater or equal to the enthalpy value at saturated temperature of $283 \mathrm{~K}$, which can be calculated directly using REFPROP.

Whereas Equation (5)

$$
h_{3} \leq 162.362 P_{3}{ }^{0.301}+100.620
$$

ensures that state 3 will always be lower or equal to the enthalpy value at saturated liquid pressure ranging from 0.1 to $0.6 \mathrm{MPa}$, where $P_{3}$ is the pressure at state 3 . The right hand side of (5) was derived using a curve fitting tool, optifit [21], that fitted a curve to a set of saturated liquid enthalpy values ranging from 0.1 to $0.6 \mathrm{MPa}$, as shown in Fig. 2. In this case, the fitting model was a power function.

4) Unit Operation Approximations: Since the rigorous unit operation functions cannot be used in this EO model, the two expressions, namely $\dot{W}_{\text {turb }}$ and $\dot{W}_{\text {pump }}$, in (3) need to be approximated as a function of enthalpy and/or pressure, hence the name approximate EO model.

The pump input power is defined in (6), where $\Delta h_{\mathrm{isen}}^{\text {pump }}$ is the isentropic pump work, $\eta_{\text {pump }}$ is the isentropic efficiency and $\dot{m}_{3}$ is the mass flow rate. Since we assume that there are no pressure drops across the heat exchangers, i.e. $P_{3}=$ $P_{2} \in[0.1,0.6] \mathrm{MPa}$ and $P_{4}=P_{1}=1 \mathrm{MPa}$, and knowing that the quality of the working fluid will always be 0 across the
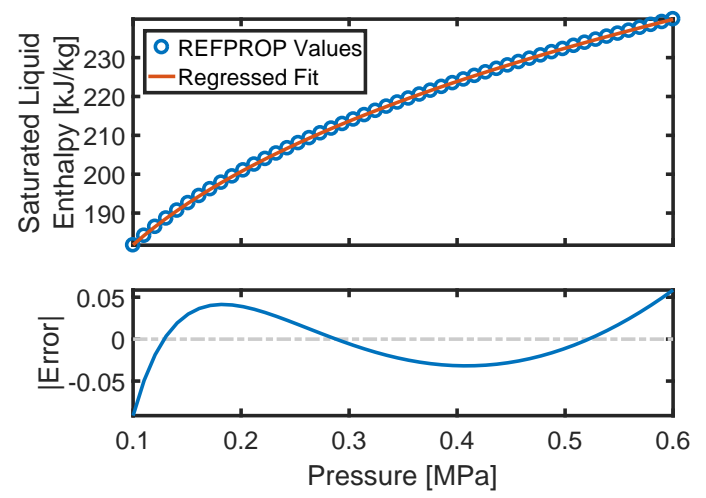

Fig. 2. Regressed fit for approximating the saturated liquid enthalpy value as a function of pressure.

pump, a set of isentropic pump work values can be calculated at various input pressures. The correlation between isentropic pump work and the inlet pressure can then be approximated via regression analysis, as shown in Fig. 3. The fitting model that was used to approximate the isentropic pump work was a quadratic polynomial curve. As a result, the pump input power now becomes

$$
\dot{W}_{\text {pump }}=\frac{\Delta h_{\text {isen }}^{\text {pump }} \dot{m}_{3}}{\eta_{\text {pump }}},
$$

where

$$
\Delta h_{\text {isen }}^{\text {pump }}=-0.271 P_{3}^{2}-0.389 P_{3}+0.626 .
$$

A similar approach can also be applied to the turbine output power; however, there are now two independent variables, namely the inlet enthalpy and the outlet pressure. Equation (9) defines the turbine output power, where $\Delta h_{\text {isen }}^{\text {turb }}$ is the isentropic turbine work, $\eta_{\text {turb }}$ is the isentropic efficiency and $\dot{m}_{1}$ is the mass flow rate. Assuming that the inlet temperature can vary between $383 \mathrm{~K}$ and the saturated vapor temperature at $1 \mathrm{MPa}$, and the outlet pressure can vary between 0.1 to $0.6 \mathrm{MPa}$, a set of isentropic turbine work values can be calculated, as shown by the black dots in Fig. 4. Since there are no explicit temperature terms in (3),

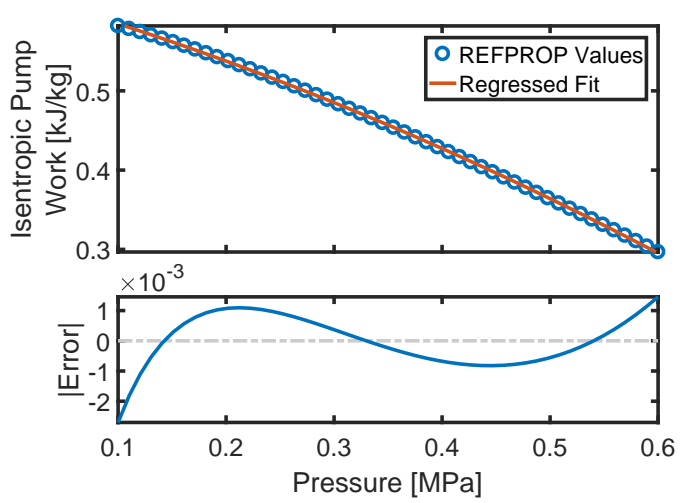

Fig. 3. Regressed fit for approximating the isentropic pump work as a function of the inlet pressure. 

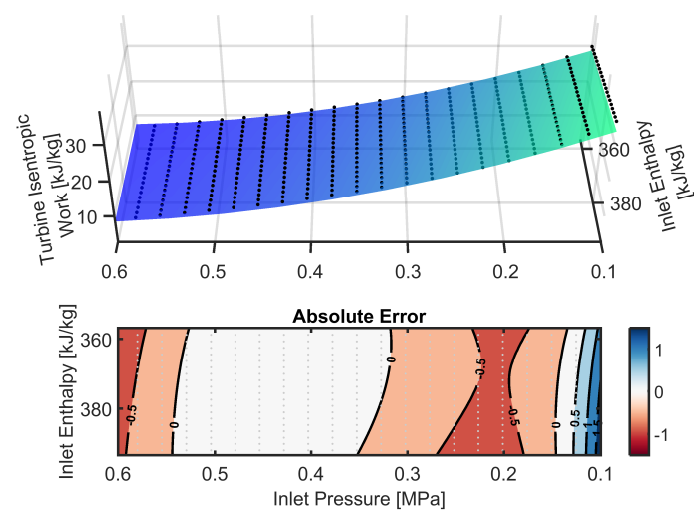

Fig. 4. Regressed fit for approximating the isentropic turbine work as a function of the inlet enthalpy and outlet pressure. (See also Fig. 5.)

the respective inlet enthalpy values were calculated for the inlet temperatures. Using a surface fitting tool, a correlation between the isentropic turbine work, the inlet enthalpy, and the outlet pressure can be approximated as a quadratic polynomial surface,

$$
\begin{aligned}
\Delta h_{\text {isen }}^{\text {turb }} & =76.439 P_{3}^{2}-0.203 P_{3} h_{1} \\
& -23.942 P_{3}+0.151 h_{1}-16.436 .
\end{aligned}
$$

So the turbine output power is now given by

$$
\dot{W}_{\text {turb }}=\Delta h_{\text {isen }}^{\text {turb }} \eta_{\text {turb }} \dot{m}_{1}
$$

5) Bounds: In order to constrain the search region and decrease the execution time, all the decision variables need to be bounded within a sensible range. For this particular optimization problem, these bounds corresponded to: 1 to $100 \mathrm{~kg} / \mathrm{s}$ for $\dot{m} ; 1$ to $500 \mathrm{~kg} / \mathrm{s}$ for $\dot{m}_{\mathrm{CW}_{\text {in }}} ; 0.1$ to $0.6 \mathrm{MPa}$ for $P_{3} ; 1$ to $1000 \mathrm{~kJ} / \mathrm{kg}$ for $h$; and $h_{g_{囚 1 \mathrm{MPa}}}$ to $h_{@ 363 \mathrm{~K}, 1 \mathrm{MPa}}$ for $h_{1}$. Once all the bounds, constraints and unit operation approximations have been established, the optimization problem can be constructed, which should follow the general nonlinear programming format.

6) EO Model Validation: In order to ensure that the approximate model is an accurate representation of the original ORC system, the optimized results were substituted into the high fidelity model (discussed in Section III-B). This validates that all our approximations are within reasonable tolerance and that the optimum results have not violated any thermodynamic laws or have not deviated too much from the actual thermodynamic properties. Table I, under the Quadratic Surface Fit column, shows the relative errors between the SM and EO models, and the average time it took for each respective optimizer to solve the optimization problem. In addition, Table I also shows the relative errors between the SM and EO models where a piecewise fit was used to approximate the turbine output power, instead of a quadratic polynomial surface fit. The rationale behind this alternative approach will be discussed in Section IV.

The discrepancies between the high fidelity SM model and the approximate EO model for all the solvers are reasonable, with the highest error being only $2.08 \%$, thus indicates that
TABLE I

THE RELATIVE ERROR [\%] BETWEEN THE APPROXIMATE EO MODEL AND THE HIGH FIDELITY SM MODEL.

\begin{tabular}{|l|c|c|c|c|c|}
\cline { 2 - 6 } \multicolumn{1}{c|}{} & \multicolumn{2}{c|}{ Quadratic Surface Fit } & \multicolumn{2}{c|}{ Piecewise Fit } \\
\hline Parameter & COBYLA & SCIP & BARON & SCIP & BARON \\
\hline$P_{3}$ & 0.00 & 0.00 & 0.00 & 0.00 & 0.00 \\
$h_{1}$ & 0.00 & 0.00 & 0.00 & 0.00 & 0.00 \\
$h_{2}$ & 0.09 & 0.06 & 0.06 & 0.01 & 0.01 \\
$h_{3}$ & 0.00 & 0.00 & 0.00 & 0.00 & 0.00 \\
$h_{4}$ & 0.00 & 0.00 & 0.00 & 0.00 & 0.00 \\
Turbine Power & 1.99 & 1.53 & 1.53 & 0.26 & 0.26 \\
Pump Power & 0.12 & 0.12 & 0.12 & 0.12 & 0.12 \\
Cooler Duty & 0.20 & 0.16 & 0.16 & 0.03 & 0.03 \\
Heater Duty & 0.00 & 0.00 & 0.00 & 0.00 & 0.00 \\
Working Fluid & 0.00 & 0.00 & 0.00 & 0.00 & 0.00 \\
Cooling Water & 0.20 & 0.16 & 0.16 & 0.03 & 0.03 \\
Thermal Efficiency & 2.08 & 1.61 & 1.61 & 0.26 & 0.27 \\
\hline Solver Time [s] & 5.53 & 14.61 & 0.91 & 20.67 & 1.08 \\
\hline
\end{tabular}

the approximate model is a fairly sensible representation of the original ORC system. However, it is possible to further improve the accuracy of the ORC model and, in some cases, reduce the complexity (order) of the fitted function by using a piecewise fit instead of a single surface fit.

\section{Piecewise Fit ApProximation}

From just visually inspecting the surface regression in Fig. 4, there are regions that the surface fit overestimates the isentropic turbine work, which can lead to large discrepancies if the plant were to operate in those regions. An alternative method is to use a piecewise fit to approximate the isentropic turbine work, as shown in Fig. 5. This allows for a more accurate fit and also could conceivably reduce the complexity of the fitting model. The aim here is to get a fit that has the lowest order (degree) possible without sacrificing the accuracy of the model. Thus, in this research, we have developed an algorithm that automatically optimizes the position of the breaks (the location where the surfaces are joined together, i.e. the solid lines in Fig. 5) in order to minimize the sum squared error (SSE) for a given number of breaks.

Just visually comparing the two figures, there is an obvious

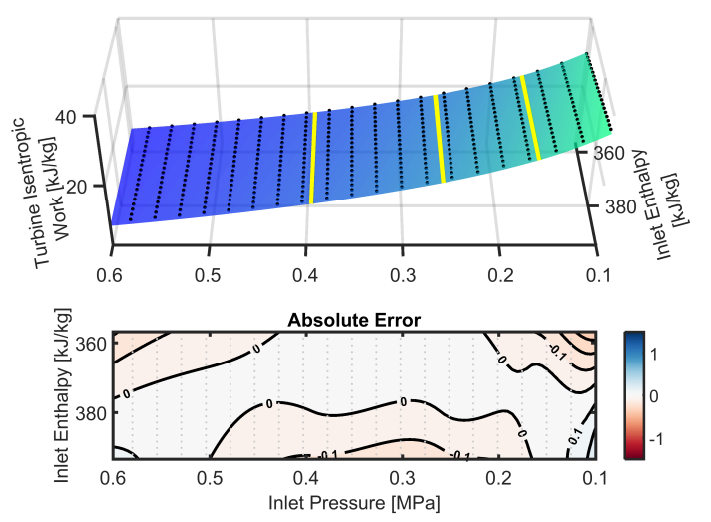

Fig. 5. Regressed fit for approximating the isentropic turbine work as a function of the inlet enthalpy and outlet pressure, using a piecewise fit. The solid lines indicate the breaks between two adjacent functions. 
accuracy improvement in using a piecewise fit. For this particular case, there are three breaks and four quadratic polynomial surface fits, which constituted to an SSE of 42.32, whereas with a single quadratic polynomial surface fitting model, the SSE was 1835.2. Note that the color axes of the error plot in Fig. 5 are deliberately set the same as for Fig. 4 to highlight the significant improvement in the accuracy of the turbine model.

Mathematically, the isentropic turbine work now becomes

$$
\Delta h_{\mathrm{isen}}^{\mathrm{turb}}= \begin{cases}\Delta h_{\mathrm{isen}}^{\mathrm{pw}}, & 0.10 \leq P_{3} \leq 0.17 \\ \Delta h_{\mathrm{isen}}^{\mathrm{pW}}, & 0.17<P_{3} \leq 0.26 \\ \Delta h_{\mathrm{isen}}^{\mathrm{pW}}, & 0.26<P_{3} \leq 0.39 \\ \Delta h_{\mathrm{isen}_{4}}^{\mathrm{pw}}, & 0.39<P_{3} \leq 0.60\end{cases}
$$

where each sub-function represents the mathematical expression for each fitted surface. For example

$$
\begin{aligned}
\Delta h_{\mathrm{isen}}^{\mathrm{pw}} & =373.431 P_{3}^{2}-0.203 P_{3} h_{1} \\
& -130.973 P_{3}+0.151 h_{1}-7.204 \\
\Delta h_{\mathrm{isen}}^{\mathrm{pw}} & =153.721 P_{3}^{2}-0.203 P_{3} h_{1} \\
& -57.527 P_{3}+0.151 h_{1}-13.342 \\
\Delta h_{\mathrm{isen}}^{\mathrm{pw}} & =67.274 P_{3}^{2}-0.203 P_{3} h_{1} \\
& -12.476 P_{3}+0.151 h_{1}-19.212 \\
\Delta h_{\text {isen }_{4}}^{\mathrm{pw}} & =31.328 P_{3}^{2}-0.203 P_{3} h_{1} \\
& +15.806 P_{3}+0.151 h_{1}-24.775
\end{aligned}
$$

In order to incorporate the piecewise fit into the optimization problem, a binary variable is added to each piecewise subfunction. However it is inefficient to multiply a binary variable with another variable because it introduces extra nonlinearity into the optimization problem. Therefore, to get around this, a modified integer programming technique from AIMMS modeling guide was implemented (in Section 7.7) [23] and applied it to this problem. This is done by introducing a new variable $y$ and equate it to the product $y_{i}=\Delta h_{\text {isen }_{i}}^{\mathrm{pw}} b_{i}$, where $b_{i}$ is the binary variable. To enforce $y_{i}$ to take the value of $\Delta h_{\mathrm{isen}_{i}}^{\mathrm{pw}} b_{i}$, the following linear constraints need to be added for each of the sub-function:

$$
\begin{aligned}
& y_{i} \leq u_{i} b_{i} \\
& y_{i} \leq \Delta h_{\text {isen }_{i}}^{\mathrm{pw}}+M\left(1-b_{i}\right) \\
& y_{i} \geq \Delta h_{\mathrm{isen}_{i}}^{\mathrm{pw}}-M\left(1-b_{i}\right) \\
& y_{i} \geq l_{i} b_{i}
\end{aligned}
$$

where $u_{i} \in\{35.03,27.58,20.90,14.49\}$ and $l_{i} \in$ $\{23.28,17.29,11.87,6.63\}$ are the sensible upper and lower bounds of $\Delta h_{\mathrm{isen}_{i}}^{\mathrm{pw}}$, and $M$ is the big-M constant that is equal to $100 u_{i}$ for this particular optimization problem. Consequently, the turbine output power now becomes

$$
\dot{W}_{\text {turb }}=\eta_{\text {turb }} \dot{m}_{1} \sum_{i=1}^{4} y_{i}
$$

In addition, respective binary variables need to be added to $P_{3}$ as well so that they can restrict the pressure value to comply with the conditions of the piecewise function in (10).

$$
\begin{aligned}
& P_{3} \geq 0.10 b_{1}+0.17 b_{2}+0.26 b_{3}+0.39 b_{4} \\
& P_{3} \leq 0.17 b_{1}+0.26 b_{2}+0.39 b_{3}+0.60 b_{4}
\end{aligned}
$$

Since only one sub-function can be selected, this can be enforced as follows

$$
b_{1}+b_{2}+b_{3}+b_{4}+b_{5}=1, \quad b_{i} \in\{0,1\}
$$

Consequently, the overall accuracy of the approximate EO model has increased significantly and has reduced the discrepancies between SM model and the EO model to no more than $0.3 \%$, as shown in Table I. However, since there are now more variables and constraints in this proposed formulation, it is reasonable to result in a longer optimization time for both SCIP and BARON. However, while this can be viewed as a disadvantage, in some cases the difference is rather small, namely BARON, and does not outweigh the significant improvement in the accuracy of the ORC model that this approach offers. Note that the optimizer COBYLA cannot be used on this problem, as it cannot solve integer programming problems, illustrating a limitation in some conventional black-box optimization schemes.

\section{RESUlts}

The approximate EO model was optimized using three different solvers, namely one black-box solver (COBYLA) and two white-box solvers (SCIP and BARON). The optimized results were validated/solved in the SM model and then compared to each other, as shown in Table II.

All three optimizers managed to find a better solution than the base case scenario, which was taken directly from [19] and solved in the SM model. Both SCIP and BARON found the highest optimum of $1063.2 \mathrm{~kW}$, whereas COBYLA converged to a local optimum of $1026.2 \mathrm{~kW}$. This constitutes to an increase of around $4.46 \%$ and $0.82 \%$ in the gross power output for the global optimum and the local optimum, respectively. Evidently, this shows a significant contribution in implementing global white-box solvers (SCIP and BARON) that can guarantee the global optimum, as opposed to a suboptimal solution obtained by black-box solvers (COBYLA).

\section{TABLE II}

COMPARISON BETWEEN THE BASE CASE AND THE OPTIMIZED EO MODEL RESULTS THAT WERE SOLVED IN THE SM MODEL.

\begin{tabular}{|l|c|c|c|c|}
\hline Variable & Base Case & COBYLA & SCIP & BARON \\
\hline$P_{3}[\mathrm{MPa}]$ & 0.2781 & 0.2782 & 0.2782 & 0.2782 \\
$h_{1}[\mathrm{~kJ} / \mathrm{kg}]$ & 393.38 & 387.12 & 356.82 & 356.82 \\
$h_{2}[\mathrm{~kJ} / \mathrm{kg}]$ & 376.59 & 370.78 & 342.82 & 342.82 \\
$h_{3}[\mathrm{~kJ} / \mathrm{kg}]$ & 211.11 & 211.11 & 211.11 & 211.11 \\
$h_{4}[\mathrm{~kJ} / \mathrm{kg}]$ & 211.77 & 211.77 & 211.77 & 211.77 \\
$\dot{m}_{1}[\mathrm{~kg} / \mathrm{s}]$ & 60.624 & 62.79 & 75.903 & 75.903 \\
$\dot{m}_{2}[\mathrm{~kg} / \mathrm{s}]$ & 60.624 & 62.79 & 75.903 & 75.903 \\
$\dot{m}_{3}[\mathrm{~kg} / \mathrm{s}]$ & 60.624 & 62.79 & 75.903 & 75.903 \\
$\dot{m}_{4}[\mathrm{~kg} / \mathrm{s}]$ & 60.624 & 62.79 & 75.903 & 75.903 \\
$\dot{m}_{\mathrm{CW}}[\mathrm{kg} / \mathrm{s}]$ & 299.09 & 298.88 & 298.04 & 298.04 \\
\hline Gross Power $[\mathrm{kW}]$ & 1017.8 & 1026.2 & 1063.2 & 1063.2 \\
\hline Solver Time $[\mathrm{s}]$ & - & 5.53 & 14.61 & 0.91 \\
\hline
\end{tabular}


While the proposed ORC system could be solved using built-in tools in commercial software, such as sequential quadratic programming (SQP) method in Aspen Plus, these conventional black-box SM (flowsheet) optimization frameworks require the derivatives of the objective function and constraints that are generally hard to obtain accurately due to the use of complex external thermodynamic packages and rigorous unit operation modules. Consequently, this can lead to inefficient optimization and convergence issues if finite difference is used to approximate the derivatives, especially for large-scale and complex systems [11]. In addition, SM optimization requires the entire flowsheet to be solved repeated, which can be significantly slower and problematic to the optimization if the flowsheet fails to converge. For this example, it took both COBYLA and fmincon relatively longer to optimize the SM model (around 7.25s and 6.51s, respectively) than to optimize the EO model (around 5.53s and $1.70 \mathrm{~s}$, respectively). Furthermore, it is important to note that while both COBYLA and fmincon did manage to find the global optimum of $1063.2 \mathrm{~kW}$ using the SM approach for this ORC system, this SM model is only restricted to black-box solvers and cannot assure global optimality.

\section{CONCLUSION}

This paper has detailed a modeling approach for ORC systems that is tailored to two advanced white-box global optimization solvers which can deterministically find the global optimum. The paper has demonstrated that both model accuracy and global optimum can be achieved by carefully approximating the output characteristics of the ORC unit operations using reasonable regression and thermodynamic analysis. The approximate EO model was optimized using three optimizers and then validated against a high fidelity model that was built using the JSteam modeling framework. As expected both SCIP and BARON found the global optimum, while COBYLA found a local optimum. Using the proposed approach to model an ORC system allowed for exact derivatives to be calculated, which aided in the accuracy of white-box optimizers in locating the global solution. In addition, the paper has shown that by using a piecewise fit, instead of a single fit function, a more accurate model approximation can be achieved without significantly compromising on the performance of the solver.

\section{ACKNOWLEDGMENT}

Financial support to this project from the Industrial Information and Control Centre, School of Engineering, Computing and Mathematical Sciences, Auckland University of Technology, New Zealand is gratefully acknowledged.

\section{REFERENCES}

[1] R. Bertani, "Geothermal power generation in the world 2005-2010 update report," Geothermics, vol. 41, no. 2012, pp. 1-29, 2012. [Online]. Available: http://dx.doi.org/10.1016/j.geothermics.2011.10. 001
[2] R. DiPippo, Geothermal Power Plants Principles, Applications, Case Studies and Environmental Impact, 4th ed. Waltham, MA: Joe Hayton: Elsevier, 2016.

[3] AspenTech, "Aspen Plus," 2015. [Online]. Available: http://www. aspentech.com/products/engineering/aspen-plus/ [Accessed: 2015-0824]

[4] Electric General, "GateCycle," 2014. [Online]. Available: https: //getotalplant.com/GateCycle/docs/GateCycle/index.html [Accessed: 2016-08-10]

[5] H. Ghasemi, M. Paci, A. Tizzanini, and A. Mitsos, "Modeling and optimization of a binary geothermal power plant," Energy, vol. 50, no. 1, pp. 412-428, 2013. [Online]. Available: http: //dx.doi.org/10.1016/j.energy.2012.10.039

[6] A. Keçeba and H. Gökgedik, "Thermodynamic evaluation of a geothermal power plant for advanced exergy analysis," Energy, vol. 88, pp. 746-755, 2015. [Online]. Available: http://dx.doi.org/10. 1016/j.energy.2015.05.094

[7] MathWorks, "fmincon," 2016. [Online]. Available: http://au. mathworks.com/help/optim/ug/fmincon.html [Accessed: 2015-12-02]

[8] A. Wächter and L. T. Biegler, "On the implementation of an interiorpoint filter line-search algorithm for large-scale nonlinear programming," Mathematical Programming, vol. 106, pp. 25-57, 2005.

[9] J. D. Currie, "Practical Applications of Industrial Optimization : From High-Speed Embedded Controllers to Large Discrete Utility Systems," PhD Thesis, Dept. Elect. Eng., AUT University, 2014.

[10] C. C. Pantelides, M. Nauta, and M. Matzopoulos, "Equation-Oriented Process Modelling Technology : Recent Advances \& Current Perspectives," in 5th Annual TRC-Idemitsu Workshop, Abu Dhabi, 2015.

[11] L. Biegler, Nonlinear Programming. Society for Industrial and Applied Mathematics, 2010. [Online]. Available: http://epubs.siam. org/doi/abs/10.1137/1.9780898719383

[12] R. Smith, Chemical Process Design and Integration, 2nd ed. West Sussex, United Kingdom: John Wiley \& Sons Inc, 2016.

[13] T. Achterberg, "SCIP: Solving constraint integer programs," Mathematical Programming Computation, vol. 1, no. 1, pp. 1-41, 2009.

[14] M. Tawarmalani and N. V. Sahinidis, "A polyhedral branch-and-cut approach to global optimization," Mathematical Programming, vol. 103, no. 2, pp. 225-249, 2005.

[15] M. J. D. Powell, A Direct Search Optimization Method That Models the Objective and Constraint Functions by Linear Interpolation. Dordrecht: Springer Netherlands, 1994, pp. 51-67.

[16] S. G. Johnson, "The NLopt nonlinear-optimization package." [Online]. Available: http://ab-initio.mit.edu/nlopt [Accessed: 2016-12-18]

[17] D. Meinel, C. Wieland, and H. Spliethoff, "Effect and comparison of different working fluids on a two-stage organic rankine cycle (ORC) concept," Applied Thermal Engineering, vol. 63, no. 1, pp. 246-253, 2014. [Online]. Available: http://dx.doi.org/10.1016/j.applthermaleng. 2013.11.016

[18] D. Budisulistyo and S. Krumdieck, "Thermodynamic and economic analysis for the pre-feasibility study of a binary geothermal power plant," Energy Conversion and Management, vol. 103, pp. 639-649, 2015. [Online]. Available: http://linkinghub.elsevier.com/retrieve/pii/ S0196890415006172

[19] A. Basaran and L. Ozgener, "Investigation of the effect of different refrigerants on performances of binary geothermal power plants," Energy Conversion and Management, vol. 76, pp. 483-498, 2013. [Online]. Available: http://dx.doi.org/10.1016/j.enconman.2013.07.058

[20] Y. A. Çengel and M. A. Boles, Thermodynamics: An Engineering Approach, 6th ed. New York: McGraw-Hill, 2008.

[21] Industrial Information \& Control Centre, "Software," 2015. [Online]. Available: http://www.i2c2.aut.ac.nz/Resources/Software. html [Accessed: 2015-09-11]

[22] National Institute of Standards and Technology, "REFPROP Version 9.1. NIST Standard Reference Database 23," 2015. [Online]. Available: http://www.nist.gov/srd/nist23.cfm [Accessed: 2016-02-05]

[23] J. Bisschop, "Integer Linear Programming Tricks," in AIMMS: Optimization Modeling. AIMMS B.V., 2016, ch. 7, pp. 75-85. 